Research Paper

\title{
The "Cavitary" Type of Angiogenesis by Gastric Cancer. Morphological Characteristics and Prognostic Value
}

\author{
Marina Senchukova ${ }^{1 凶}$, Mikhail V. Kiselevsky² \\ 1. Orenburg State Medical Academy, Orenburg, Russia \\ 2. Institute of Experimental Diagnostics and Therapy of Tumors, Russian Oncological Scientific Center named after N.N.Blokhin, Moscow, \\ Russia
}

\begin{abstract}
$\triangle$ Corresponding author: Marina Senchukova, PhD in Medicine, Associate Professor of Oncology, The Orenburg State Medical Academy, 460000, Russia, Orenburg, Sovetskaya st., 6. (Tel) +7 (3532) 776103 (Fax) +7 (3532) 772 459. masenchukova@yandex.com

() Ivyspring International Publisher. This is an open-access article distributed under the terms of the Creative Commons License (http://creativecommons.org/ licenses/by-nc-nd/3.0/). Reproduction is permitted for personal, noncommercial use, provided that the article is in whole, unmodified, and properly cited.
\end{abstract}

Received: 2014.01.29; Accepted: 2014.03.02; Published: 2014.03.20

\begin{abstract}
The "cavitary" type of angiogenesis in patients with gastric cancer (GC) is described for the first time. Material and methods: The samples of tumour and adjacent gastric mucosa (GM) in 73 patients with GC who had undergone radical surgery were being studied. The sections were stained with hematoxylin and eosin (H\&E) and immunohistochemically (IGH) using antibodies to CD34. Results: A new type of vessel formation consists of the appearance of cavitary structures (CS) in tumours and the adjacent GM, which are then lined by endothelial cells and merged into the blood vessels of the organ. We believe that the CS can be formed by means: I) of the abruption of layers of epithelial cells (both normal and tumoral) from their underlying foundation and their desquamation into the lumen of the "obliterated" gastric glands (GG); 2) of the dilatation of the GG and thinning of their walls; 3 ) of the formation of "cavity" directly in the lamina propria of GM or in the tumoral stroma. It was noted that only the presence of multiple "cavitary" vessels $(\mathrm{CV})$ of type-I had been associated with the decrease of 3 -year overall survival $(O R=15,0$, $95 \% \mathrm{Cl}=2,96-76,3 \mathrm{I})$ and relapse-free survival $(\mathrm{OR}=\mid 4,93,95 \% \mathrm{Cl}=4,34-5 \mathrm{I}, 38)$. We also observed the improvement of the long-term outcomes in patients with GC having received antibacterial therapy (AT) before surgery that can be associated with its influence on the formation of CV type-I. Conclusion: The described new type of angiogenesis is of great clinical importance.
\end{abstract}

Key words: gastric cancer, angiogenesis, vessels morphology, antibacterial therapy, clinical significance

\section{Introduction}

The key role of angiogenesis in the progression of malignant neoplasms has been shown in a large number of studies [1-3]. However, the conducted researches allowed to find that the tumoral vasculature is non-homogeneous in its structure, the degree of maturity of the vessels and their origin. To date, several types of blood vessel formation by tumoral growth are known: sprouting angiogenesis [4-5], vessel co-option [6], vasculogenic mimicry [7-8], intussusceptive angiogenesis [9], and vasculogenesis involving progenitor cells [10-11].

In studies of the features of angiogenesis in pa- tients with GC we encountered an interesting phenomenon which was considered as a new, previously undocumented type of vessel formation. According to it, the aim of the present study was to give a morphological characteristics and a clinical evaluation of this new type of vessel formation GC.

\section{Material and methods}

\section{Patients}

74 patients with GC who had undergone radical surgery (R0) in the Orenburg Regional Clinical On- 
cology Center between January 2009 and July 2010 were included in this prospective study. There were 43 men $(58.9 \%)$ and 30 women $(41.1 \%)$. The average age of the patients was 61.2 \pm 9.3 years (from 34 to 78 years, the median - was 61 years). The involvement of the upper third of the stomach was in 14 patients $(19.2 \%)$, the middle third - in 18 patients $(24.7 \%)$, the lower third - in 39 patients (53.4\%), and the total gastric cancer was in 2 patients $(2.7 \%)$. In accordance with the TNM classification of malignant tumours [12] the distribution of patients according to the stages of the disease was as follows: T1-2N0M0 was in 33 patients $(44,2 \%), \mathrm{T} 3 \mathrm{~N} 0 \mathrm{M} 0$ - in $10(13.7 \%)$ and T2-4N1-2M0 - in $30(41.1 \%)$. Intestinal and diffuse-types of GC were in 41 cases $(56.2 \%)$ and 32 cases $(43.8 \%)$ respectively. Tumours were good-differentiated (G1) in 27 patients (36.9\%), moderate (G2) - in $14(19.3 \%)$, and poor (G3) in $9(12.3 \%)$, and 23 patients $(31.5 \%)$ had signet ring cell carcinoma. Subtotal distal resection was performed in 56 cases $(76.7 \%)$, subtotal proximal resection - in 10 cases $(13.7 \%)$, and gastrectomy - in 7 cases $(9.5 \%)$. D2 volume lymphadenectomy was performed in all patients, with D3 elements - in 38 patients $(52.0 \%)$.

28 of the 73 patients (38.4\%) have received AT before the surgery apropos of the preliminary diagnosis of gastritis or stomach ulcer. The presence of AT was taken into account only if the treatment had been in a period of 1 to 1.5 months before surgery, duration of not less than 7 days, with the appointment of 2 or more antibiotics. The patients received most commonly a combination of the following drugs: clarithromycin + amoxicillin, amoxicillin + clarithromycin + metronidazole, amoxicillin + metronidazole.

Patients with decompensation of chronic diseases, acute infection pathology, severe allergic processes were not included in the study as well as the ones who received corticosteroids, antihistamines, non-steroidal anti-inflammatory drugs and neoadjuvant chemotherapy.

\section{Histological analysis}

After removal of stomach (within 30 minutes) a greater curvature of the organ was opened and biopsy samples were taken from the tumour and the adjacent macroscopically non-tumorous mucosa at a distance of 3-5 cm from the tumour margin. Taken specimens were fixed in buffered formalin, embedded in paraffin. $4 \mu \mathrm{m}$ thick slices were stained with Mayer's hematoxylin and eosin. Histological slides were studied by light microscopy (Optika B-350 microscope, connected to a ScopeTek DCM500 camera, Italy). To suit the Updated Sydney System`s requirements, the degree of atrophy, intestinal metaplasia and dysplasia were evaluated as "none", "mild", "moderate" and "severe". The severity of polymorphonuclear cell infiltration (PCI) was calculated as density of cells on area unit equal to $0.42 \times 0.28 \mathrm{~mm}^{2}$. The number of lymphoid follicles (LF) and "cavitary" type vessels in the GM and tumour was assessed by visual analog way (none, single - no more than two in the field of view, and multiple - more than two in the field of view). The histological type of GC was assessed according to the Lauren's classification.

\section{Immunohistochemistry}

The sections were dewaxed and rehydrated by sequential immersion in xylene and graded ethanol and water. For antigen retrieval, the sections boiling for $10 \mathrm{~min}$ in citrate buffer $(\mathrm{pH} \mathrm{6)}$ and endogenous peroxidase activity was blocked with $30 \mathrm{~mL} / \mathrm{L}$ hydrogen peroxide solution. Adjacent slides were incubated with Anti-CD34 (QB-END/10, Novocastra Laboratories Ltd) monoclonal antibodies in diluted at 1:50 for $1 \mathrm{~h}$ at room temperature. The visualization system has included DAB and Hematoxylin counterstaining. For negative control sections, primary antibody was replaced with phosphate-buffered saline and processed in the same manner.

The obtained data were compared with clinical features of GC: stage, sizes, localization, histological type of tumour, the presence of AT in the anamnesis and 3-year overall survival (OS) and relapse-free survival (RFS).

\section{Statistics}

Statistical analysis was performed using the Statistica 6.0 software. The correlations between different data were evaluated using nonparametric Spearman's rank correlation or gamma correlation. Chi-square tests were carried out to analyze the difference of distribution among the categorized data. The survival was analyzed by the Kaplan-Meier method. The log-rank test was used to compare survival curves between subgroups of patients. Cox's proportional hazards model was used for multivariate analysis of prognostic factors. The association between the 3-year survival (OS and RFS) and both the number of "cavitary" vessels in tumour and the presence of AT in anamnesis was estimated using an odds ratio (OR) and a $95 \%$ confidence interval $(\mathrm{CI})$. A value of $\mathrm{P}<0.05$ was considered statistically significant.

\section{Results}

The performed study allowed us to establish a previously undocumented type of vessel formation by GC. It has been named as "cavitary type of neoangiogenesis" based on the general peculiarities of the vessel formation. They consist in the formation of cavitary structures (CS) in tumour and the adjacent 
GM (1 stage), which are then lined by endothelial cells (2 stage) and merged into blood vessels of the organ (3 stage). We believe that there are three different types of the formation of the described $\mathrm{CV}$ :

\section{CV type-I}

The first type of $\mathrm{CV}$ formation is associated with the abruption of layers of epithelial cells from their underlying foundation and their desquamation into the lumen of the "obliterated" gastric or tumour glands. The formation of CS type-1 can be observed in the areas of GG located adjacent to the tumour of the GM (Fig. 1A), cancerous glands (Fig. 1B) and discrete groups of tumour cells (Fig. 1C). By IHC staining we can observe that the internal surface of the described CS can be fully or partially lined by endothelial cells expressing CD34 (Fig. 1D). The cytoplasm of these cells is being stained unevenly with the marker and has an uneven surface with a number of protuberances. Sometimes it seems that the inner lining of the CS appears very early, while the desquamated cell masses are still contained in their lumen (Fig. 1C). In this case the cytoplasm of the lining cells does not express CD34 and is difficult to be distinguished on the light-optical level. The cells with large, light, oval-shaped nuclei are sometimes observed in the structure of such endothelial lining. The CS and a newly formed CV type-1 can have a different shape, generally the same as the gastric glands next to them have got (Fig. 1E). In the lumen of the CV type- 1 tumoral and epithelial emboli are often detected (Fig. $1 \mathrm{~F})$.
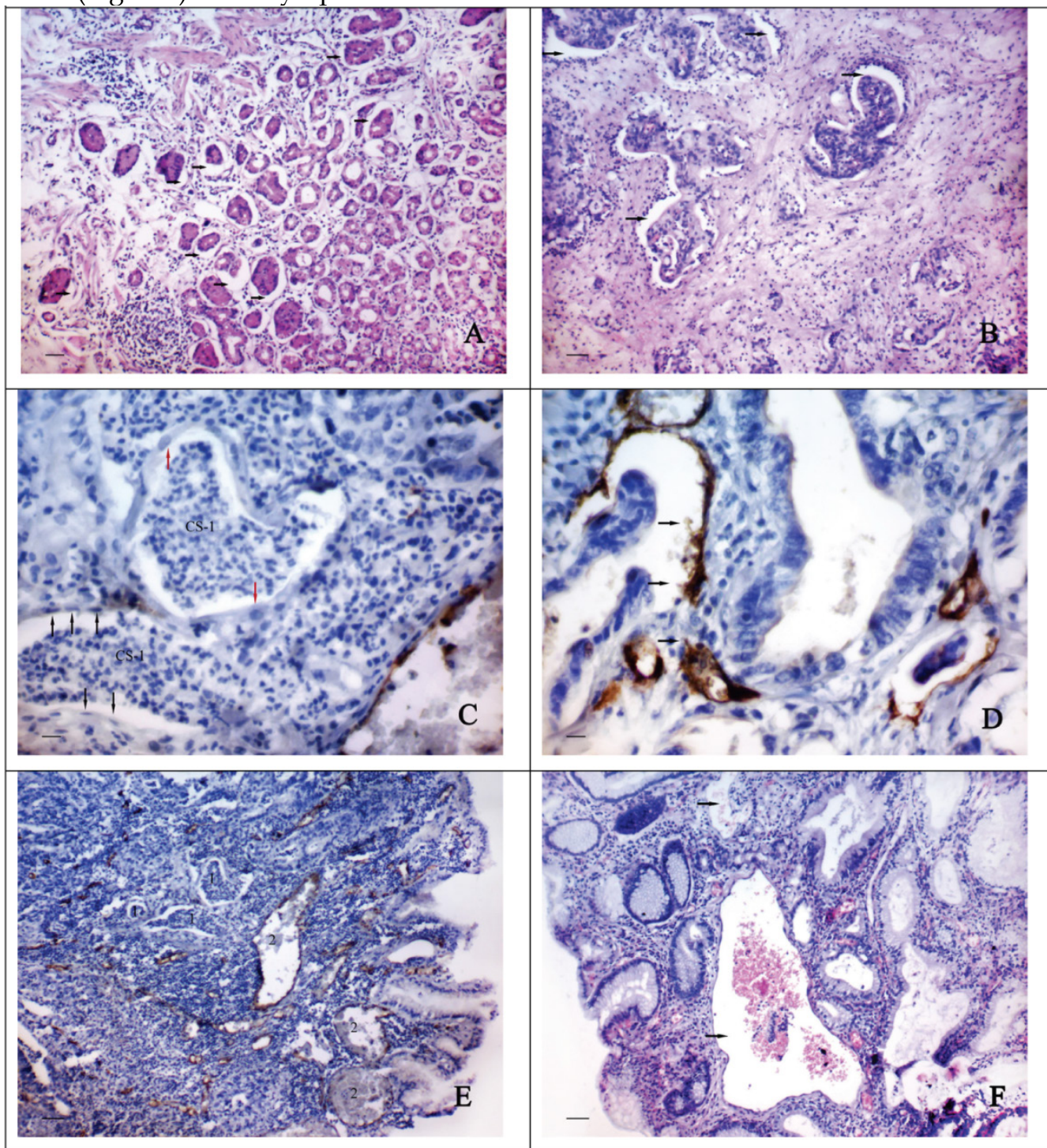

Figure I. The morphological features of "cavitary" vessels of type-I. A shows the abruption of layers of epithelial cells from their underlying foundation (arrows) and their desquamation into the lumen of the gastric glands and tumour glands (B): H\&E stain, bars $=100 \mu \mathrm{m}$; $\mathbf{C}$ - the "cavitary" structures (CS- $I$ ) in area of the discrete groups of tumour cells. The cytoplasm of the lining cells (black arrows) does not express CD34. The some cells of endothelial lining have large, light, oval-shaped nuclei (red arrows): immunoperoxidase staining, bars $=20 \mu \mathrm{m}$; D - the "cavitary" structures in tumour stroma with a partial endothelial lining, cells of which express CD34. The cytoplasm of these cells has an uneven surface with a number of protuberances (arrows): immunoperoxidase staining, bars $=20 \mu \mathrm{m}$; $\mathbf{E}-$ the "cavitary" structures (I) and the vessels of type-I (2) in tumour stroma having different shapes and sizes: immunoperoxidase staining, bars $=100 \mu \mathrm{m} ; \mathbf{F}$ - the "cavitary" vessels 0 type- $I$ (arrows) with the epithelial emboli in their lumen: H\&E stain, bars $=100 \mu \mathrm{m}$. 


\section{CV type-2}

The second type of $\mathrm{CV}$ formation is associated with the dilatation of normal or tumoral glands and the thinning of their walls. In this case the height reduction of the epithelial cells and their gradual flattening are observed (Fig. 2A). The lumen of the dilatated GG often contains a characteristic secret made up of leukocytes and eosinophilic structureless masses. Sometimes abnormal large cells (up to 30-45 $\mu \mathrm{m}$ ) having a different shape with strongly eosinophilic cytoplasm and a small hyperchromatic nucleus can be seen in it. These cells are also found in the lamina propria of the GM and on the surface of epithelial cells (Fig. 2B). The thinning of the walls of the GG possibly leads to their purely mechanical confluence with the intimately adjacent blood vessels. Perhaps, in this case the endothelial lining may be formed over the epithelial cells since double lining is sometimes seen in vessels of this type (Fig. 2A). The vessels of such type often have an irregular, bizarre shape, similar to that of the adjacent GG. In some cases in one slide there can be observed the formation of the vessels both of the first type and the second one (Fig. 2C).

We suppose that sometimes the formation of $\mathrm{CV}$ type-2 can occur without the dilatation of the gastric glands. In this case size, shape and localization of such vessels geometrically conform to the abovementioned parameters of the GG located near (Fig. 2D). The cells lining the described vessels have round or oval-shaped nuclei, often hyperchromatic with the presence of polychromasia. Sometimes above the described cells the cells with flattened, elongated nuclei, possibly endothelial ones can be observed.

\section{CV type-3}

The third type is associated with the formation of CS directly into the lamina propria of the GM or the tumour stroma. This supposition is due to the fact that in the some cases we observed a characteristic cellular structure of the connective tissue of the lamina propria of GM (Fig. 3A), often combining with the expressed phenomena of diapedesis of erythrocytes and associating with a number of clinically relevant factors. Most often than not the described CS were observed in the GM at the level of gastric pits or directly in the stroma bordering upon tumour tissue. Sometimes the cavities with endothelial lining were revealed. The cytoplasm of the cells of such lining weakly expressed CD34 and was characterized by the presence of a number of protuberances and intracavitary growths (Fig. 3B).

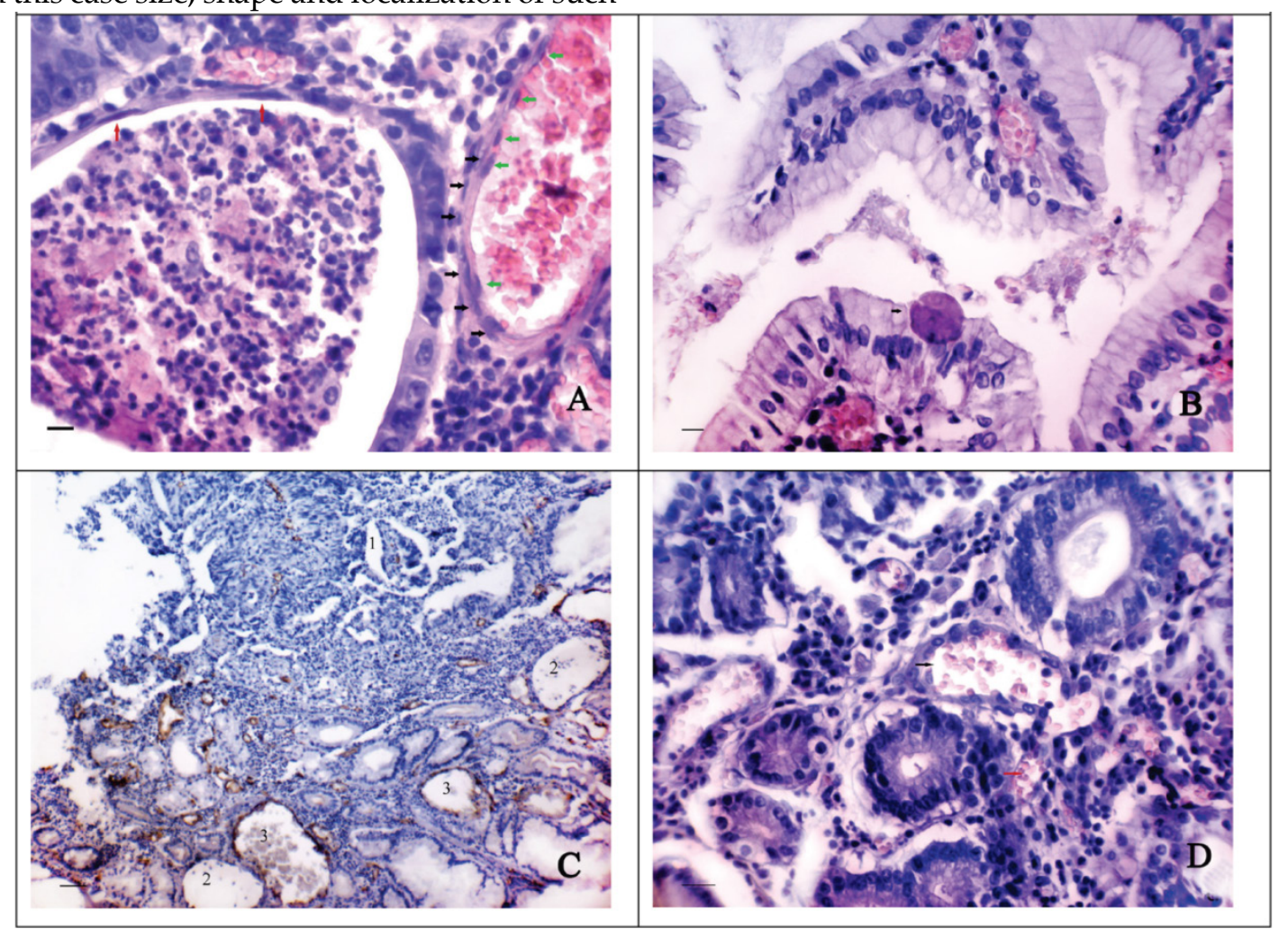

Figure 2. The morphological features of "cavitary" vessels of type-2. A shows the reduction of the epithelial cells height and their flattening (red arrows) in the dilated tumoral gland. The lumen of gland contains the characteristic secret made up of leukocytes and eosinophilic structureless masses. The adjacent "cavitary" vessel of type-2 has the inner (black arrows) and the outer (green arrows) lining: H\&E stain, Bars $=20 \mu \mathrm{m}$; B - the abnormal large cell ( $46 \mu \mathrm{m})$ with strongly eosinophilic cytoplasm and a small hyperchromatic nucleus is located on the surface of epithelial cells: H\&E stain, Bars $=20 \mu \mathrm{m} ; \mathbf{C}$ - the "cavitary" structures of type-I (I), the dilated gastric glands (2) and the "cavitary" vessels of type-2 (3) in the stroma bordering upon tumour tissue: immunoperoxidase staining with the anti-CD34 monoclonal antibody, bars = $100 \mu$ m; $\mathbf{D}$ shows the confluence of the "cavitary" vessel of type-2 (black arrow) and the adjacent blood vessels (red arrow). The cells lining the "cavitary" vessel have oval-shaped hyperchromatic nuclei with the presence of polychromasia: H\&E stain, bars $=30 \mu \mathrm{m}$. 

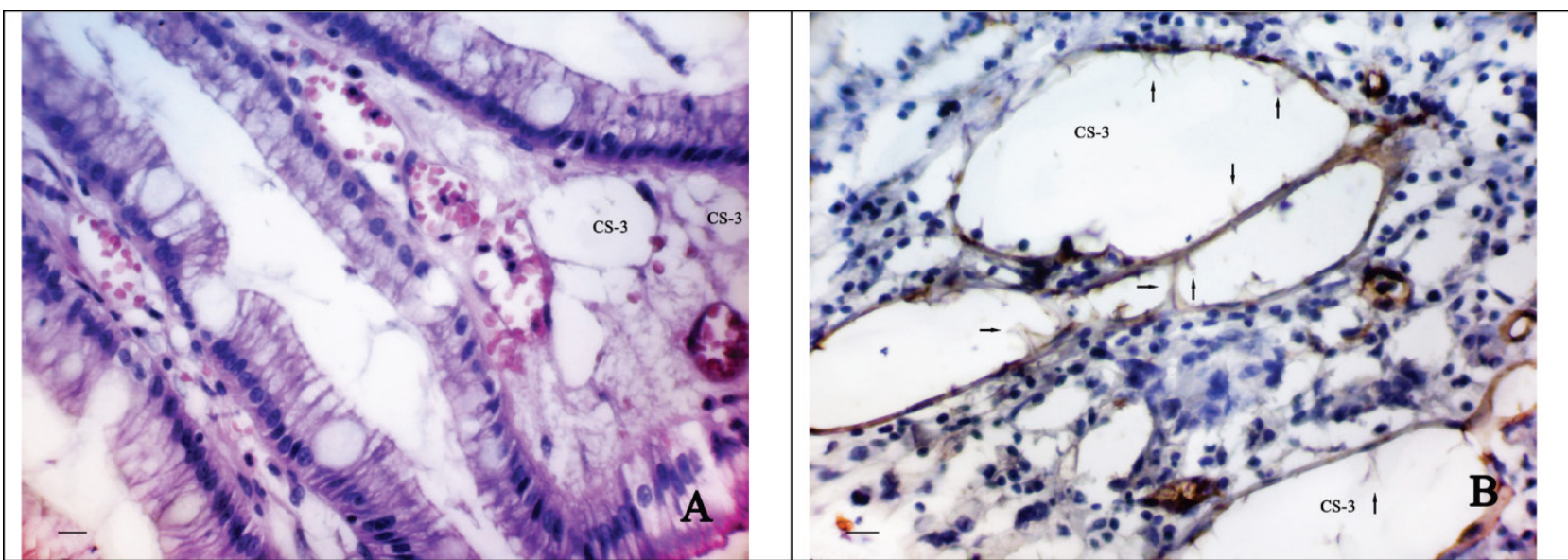

Figure 3. The morphological features of "cavitary" vessels of type-3. A shows the "cavitary" structures (CS-3) in the lamina propria of the gastric mucosa at the level of gastric pits: H\&E stain, bars $=20 \mu \mathrm{m}$; B - the cytoplasm of cells lining the "cavitary" structures (CS-3) weakly expresses CD34 and has a number of protuberances and intracavitary growths (arrow): immunoperoxidase staining with the anti-CD34 monoclonal antibody, bars $=20 \mu \mathrm{m}$.

\section{Clinical significance of "cavitary" type of angiogenesis}

The Spearman rank correlation analysis $(\rho)$ and gamma correlation coefficient test (gamma) showed that the number of CV type- 1 correlated with sizes $(\rho=0,316, t=2,81, p=0,006)$, histological type (gam$\mathrm{ma}=0,344, \mathrm{z}=2,51, \mathrm{p}=0,01)$, degree of tumour differentiation (gamma $=0,318, z=2,79, p=0,005$ ), stage (gam$\mathrm{ma}=0,524, \mathrm{z}=4,27, \mathrm{p}=0,00001$ ), nodal stage (gam$\mathrm{ma}=0,520, \mathrm{z}=4,19, \mathrm{p}=0,00003)$, three-year overall survival rate (OS) (gamma $=-0,778, z=-4,64, p<0,00001$ ) and Relapse-free survival (RFS) (gamma $=-0,766$, $\mathrm{z}=-5,81, \mathrm{p}<0,00001)$. Also the correlation between the number of $\mathrm{CV}$ type- 1 and $\mathrm{CV}$ type-3 was noted (gamma $=0,577, \mathrm{z}=4,47, \mathrm{p}<0,00001$ ).

In the cases with multiple CV type- 1 compared to the cases without them or when they were single, the size of the primary tumour was significantly larger (Fig.4) and diffuse type, poor differential forms of GC, advanced stage and nodal stage N2 more frequently occurred (Table 1). 3-year OS and RFS were practically identical if the CV type- 1 in tumour stroma were single or absent, and significantly worse when the CV type- 1 were multiple (Fig. 5A-B). With or without the multiple CV type-1, the 3-year OS was $52.7 \%$ and $93.9 \%$ respectively $(\mathrm{t}=0,0013, \mathrm{OR}=15,0,95 \%$ $\mathrm{CI}=2,96-76,31$ ), and the RFS was $32.4 \%$ and $87.7 \%$ respectively $(\mathrm{t}=0,0001, \mathrm{OR}=14,93,95 \% \mathrm{CI}=4,34-51,38)$.

The presence of $\mathrm{CV}$ type-2 correlated only with the degree of GM metaplasia (gamma $=0,580, t=3,18$, $\mathrm{p}=0,002)$. For mild degree of metaplasia the CV type- 2 were absent, single or multiple in $88.2 \%, 11.8 \%$ and $0 \%$, respectively, whereas at moderate or marked degree of metaplasia - in $67.6 \%, 18.9 \%$ and $13.5 \%$, respectively $\left(x^{2}=6,15, p=0,046\right)$.
Table I. Frequency of CV type-I according to the clinical characteristics

\begin{tabular}{|c|c|c|c|c|c|c|c|}
\hline \multirow[t]{3}{*}{ Clinical characteristics } & \multicolumn{6}{|c|}{ Number of CV type-1 } & \multirow{3}{*}{$\begin{array}{l}\text { Chi-squared test } \\
\text { (two-sided) }\end{array}$} \\
\hline & \multicolumn{2}{|c|}{ none } & \multicolumn{2}{|c|}{ single } & \multicolumn{2}{|c|}{ multiple } & \\
\hline & $\mathrm{n}$ & $\%$ & $\mathrm{n}$ & $\%$ & $\mathrm{n}$ & $\%$ & \\
\hline \multicolumn{8}{|c|}{ Lauren predominant type } \\
\hline Intestinal & 20 & 48,8 & & 26,8 & 10 & 24,4 & $x^{2}=3,42$ \\
\hline Diffuse & 10 & 31,2 & & 25,0 & 14 & 43,7 & $\mathrm{p}=0,18$ \\
\hline \multicolumn{8}{|l|}{ Differentiation } \\
\hline G1 & 16 & 59,3 & & 25,9 & 7 & 14,8 & $x^{2}=9,64$ \\
\hline G2 & 4 & 28,6 & & 28,6 & 6 & 42,9 & $\mathrm{p}=0,14$ \\
\hline G3 & 2 & 25,0 & & 12,5 & 5 & 62,5 & \\
\hline Ring cell carcinoma & 8 & 33,3 & & 29,2 & 9 & 37,5 & \\
\hline \multicolumn{8}{|l|}{ Stage } \\
\hline T1-2N0M0 & 22 & 51,2 & 13 & 30,2 & 8 & 18,6 & $x^{2}=13,01$ \\
\hline T3N0M0 & 4 & 44,4 & 2 & 22,2 & 3 & 33,3 & $\mathrm{p}=0,01$ \\
\hline $\mathrm{T} 2-4 \mathrm{~N} 1-2 \mathrm{M} 0$ & 4 & 19,1 & 4 & 19,1 & 13 & 61,8 & \\
\hline \multicolumn{8}{|l|}{ Nodal stage } \\
\hline No & 19 & 57,6 & 10 & 30,3 & 4 & 12,1 & $x^{2}=12,35$ \\
\hline N1 & 3 & 30,0 & 3 & 30,0 & 4 & 40,0 & $\mathrm{p}=0,01$ \\
\hline N2 & 8 & 26,7 & 6 & 20,0 & 16 & 53,3 & \\
\hline
\end{tabular}

In its turn, the CV type- 3 correlated only with the histological type of tumour (gamma=0,403, $z=2,68$, $\mathrm{p}=0,008)$. By the diffuse and intestinal type of GC they were revealed in $55,9 \%$ and $44,1 \%$ cases respectively $\left(x^{2}=3,24, p=0,07\right)$.

The multivariate Cox proportional hazard regression analysis indicated that TNM stage $(p=0,003)$, nodal stage $(p=0,013)$, the number of CV type- 1 $(p=0,005)$ and the presence of AT in the anamnesis as to the preliminary diagnosis of gastritis or stomach ulcer $(p=0,001)$ were significantly independent prognostic factors in patients with GC. It was established that the presence of AT in anamnesis significantly correlated with 3-year OS (gamma $=0,727, z=4,27$, $p=0,00002)$ and RFS (gamma $=0,987, \quad z=4,65$, $\mathrm{p}=0,000003$ ). The 3 -year OS rate was $100 \%$ and $67.8 \%$ respectively $(\mathrm{t}=0,0014, \mathrm{OR}=0,15,95 \% \mathrm{CI}=0,04-0,58)$, 
and the RFS rate was $89.5 \%$ and $56.3 \% \quad(t=0,002$, $\mathrm{OR}=0,08,95 \% \mathrm{CI}=0,01-0,67)$ respectively in patients who have received and not received AT (Fig. 6A-B) Still more differences in survival rate were observed in a group of patients with local advanced GC (stages
T3N0M0-T2-4N1-2M0). In this group the 3-year OS rate was $100 \%$ and $57,4 \% \quad(p=0,003, \quad O R=0,14$, $\mathrm{CI}=0,03-0,65)$ and the RFS was $78.0 \%$ and $34.6 \%$ $(\mathrm{p}=0,005, \mathrm{OR}=0,097, \mathrm{CI}=0,01-0,85)$ with or without AT in anamnesis respectively (Fig. 7A-B).

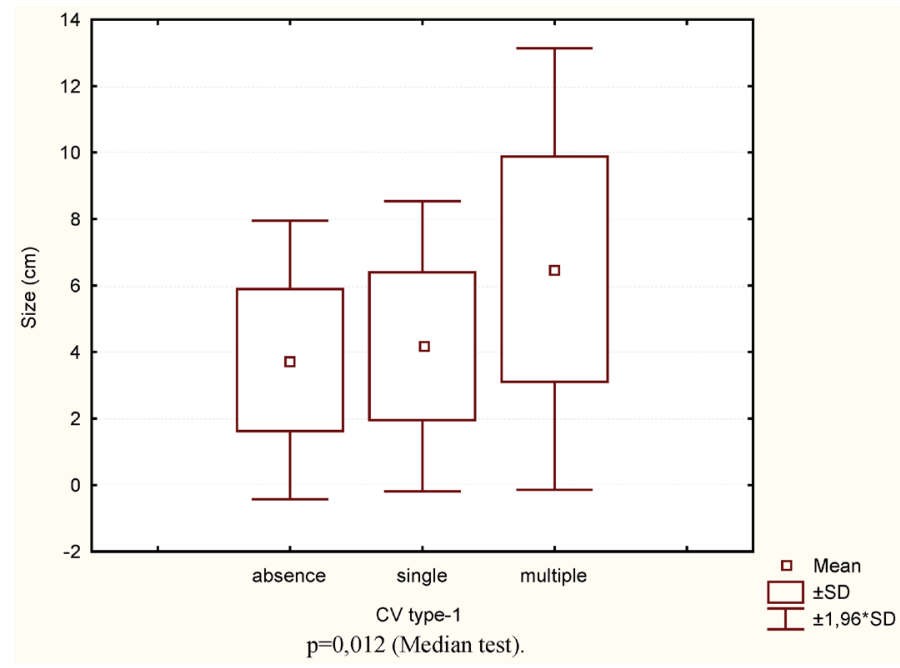

Figure 4. The tumour sizes according to the number of CV type-I.
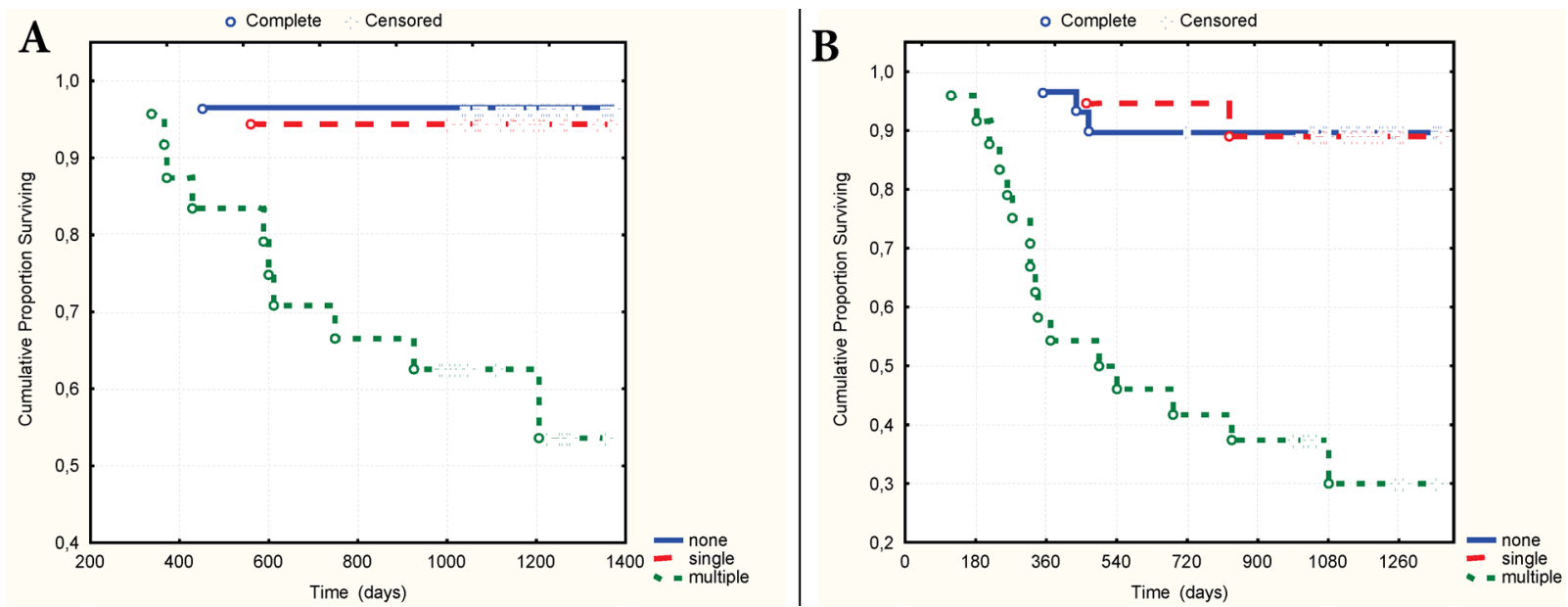

Figure 5. A. 3-year overall surviving according to the number of CV type-I. B. 3-year relapse-free surviving according to the number of CV type-I.
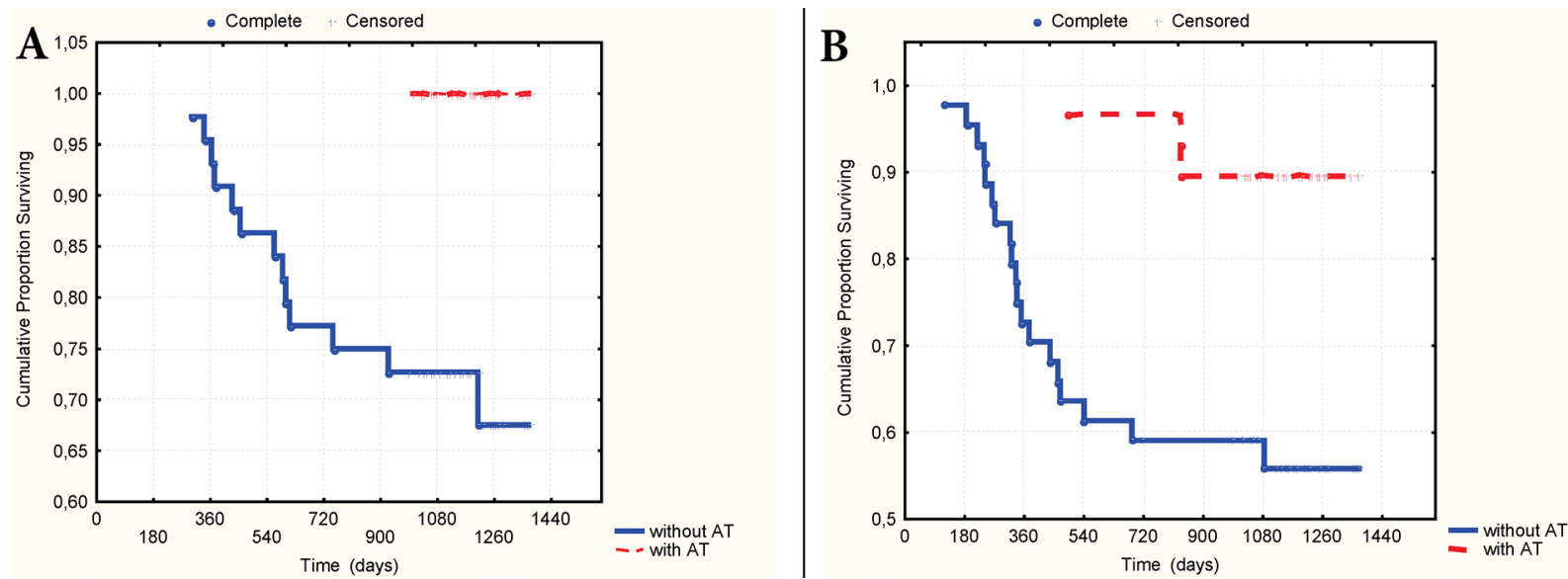

Figure 6. A. 3-year overall surviving according to the presence of AT in anamnesis. B. 3-year relapse-free surviving according to the presence of AT in anamnesis. 

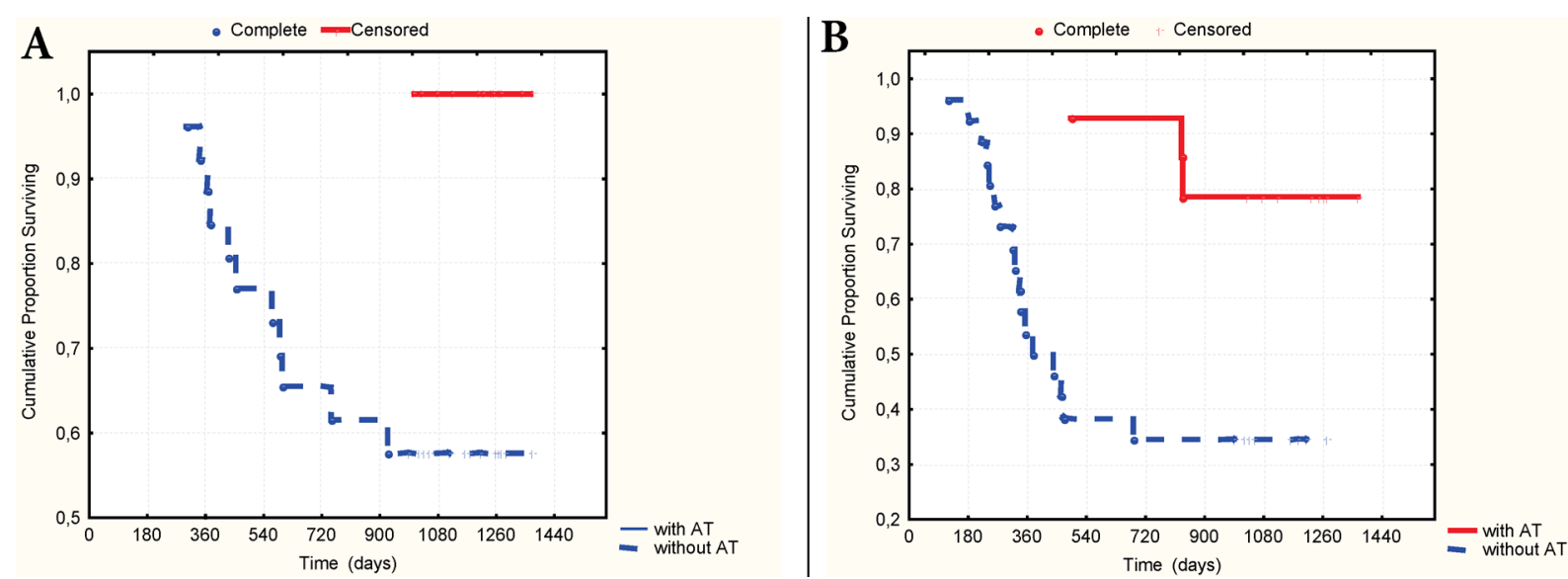

Figure 7. A. 3-year overall surviving according to the presence of AT in anamnesis in a group of patients with stages T3N0 - T2-4NI-2. B. 3-year relapse-free surviving according to the presence of AT in anamnesis in a group of patients with stages T3N0 - T2-4NI-2.

It is important to note that the recurrence of the disease during of the first year after surgery occurred in 12 of 44 patients, who had not received AT (60\% of all cases of relapse in this group). In the group of patients having received AT there was no cases of relapse during this period. It was also found that the level of polymorpho-cellular infiltration of GM was below in patients with advanced GC having received AT $(8.75+3.56$ and $10.88+3.64$ cells on the conditional area unit in patients receiving and not receiving AT respectively, $\mathrm{p}=0.03$ ) and the multiple $\mathrm{CV}$ type- 1 were being detected more rarely (in $18,2 \%$ and $58,3 \%$ respectively, $\mathrm{p}=0,02)$ as well.

\section{Discussion}

A large number of studies testify that the vasculature in a tumour is heterogeneous in a structure and different types of vessels may respond differently to the various effects including the use of drugs [4, 6-7]. This fact can be a main reason that the results of the human clinical trials with a wide variety of angiogenesis inhibitors to treat of malignant neoplasm have been very disappointing. In view of these data the relevance of new researches for understanding of mechanisms of tumoral vessels formation does not cause doubts.

In the present study we are describing a new way of angiogenesis characterized by the formation of the CS in the tumour stroma and adjacent GM, then being lined by endothelial cells and merged into the blood vessels of the organ. The analysis of the obtained data allowed us to propose the existence of three possible variants of the formation of such CS. The first one is associated with the abruption of layers of epithelial cells from their underlying foundation and their desquamation into the lumen of the "obliterated" GG or tumour glands, the second - with the dilatation and thinning of the walls of GG, and the third one is associated with the formation of CS di- rectly in the lamina propria of the GM or the tumour stroma. It is conceivable that the important role in the formation of the cavitary type of vessels can play inflammatory changes in the tumour stroma and the adjacent GM. It is known that an active inflammatory process is connected with the increased secretion by immune cells of cytokines, chemokines, growth factors and proteases [13-14] that promote the activation of tumour angiogenesis on the one hand [15-16], influence the adhesive properties of tumour cells on the other [17-18]. Besides, the some studies have shown that the immune cells may be directly associated with tumor progression and invasion [19-22].

It can be predicted with certainty that the previously described "Retraction Artifact" (it is a space between tumour cells and their surrounding stroma) has a direct relationship to the type-1 of "cavitary" angiogenesis. The prognostic significance of "Retraction Artifact" as a factor associated with tumour progression was mentioned in a number of studies [23-25]. However, in contrast to the cited sources, we succeeded to link this phenomenon to a previously undocumented type of angiogenesis and to show that tumoral emboli in blood vessels can be formed at the expense of the abruption of tumour cells from their adjacent stroma and of their desquamation directly into the lumen of the vessel of "cavitary" type. The morphological features of the CV type- 1 testify that the disorder of the adhesive properties of tumour cells is of the key importance in their formation. The focal disruptions in the tumor capsule [26-27] associated with increased immune cell infiltration [19-20] can have also attitude to the formation of the CV type-1.

It is possible that another important factor associated with the formation of CV type- 1 and type- 3 is a phenomenon of the increased vascular permeability that on the one hand may influence the process of stroma retraction and on the other - promote the formation of a fibrin matrix and a migration of endothe- 
lial cells [28]. There is a good reason to believe that the parallel processes of formation and lysis of stroma that more actively occurring at the boundary between tumour and adjacent tissue may contribute to the formation of CV type-3. Taking into account the similarity in the morphology of cells lining CV type- 1 and CV type-3 (the absence or weak expression of CD34, an uneven surface with a number of protuberances and intracavitary growths), it is impossible to exclude their common origin and the participation of progenitor cells in this type of angiogenesis. Their increase was noted in many malignant tumours [10-11].

When considering the role of angiogenesis in the progression of GC it is important to bear in mind that the surgery itself contributes to tumour progression through the activation of inflammation and neoangiogenesis factors [2, 29]. Perhaps a large number of relapses during the first year after surgery among the patients having not received AT is attributable to this fact. It can be assumed that the administration of AT before surgery contributes to the reduction of the inflammatory process activity and normalization of the adhesive properties of tumour cells, which in turn decreases metastasis risk and improves the long-term results of the treatment of GC. The decrease in the density of PCI in the GM and the reduction of the number of cases with multiple CV type- 1 in the tumour stroma in patients after receiving the AT testify in favour of this hypothesis, as well as the data literature on the improvement of the long-term results of malignant tumours treatment when using antibacterial drugs [30-32].

\section{Summary}

Thus, the results of the current study testify that the described "cavitary" type of angiogenesis possibly play a key role in the progression of GC. This follows from the fact that the presence of multiple CV type-1 is closely associated with the presence of metastases in RLN and the decrease of RFS and OS of patients with GC. It is conceivable that a violation of the adhesive properties of tumour cells, inflammatory changes in the tumour stroma and the adjacent GM and microvascular hyperpermeability may be associated with the formation of CV type- 1 . We believe that further studies should be carried out to investigate the mechanisms of "cavitary" type of neoangiogenesis and the role of AT in the treatment of malignant tumours.

\section{Abbreviations}

AT: antibacterial therapy; CI: confidence interval; CS: cavitary structures; CV: "cavitary" vessels; GC: gastric cancer; GG: gastric glands; GM: gastric mucosa; H\&E: hematoxylin and eosin; IGH: im- munohistochemically; LF: lymphoid follicles; OR: odds ratio; OS: overall survival; PCI: polymorphonuclear cell infiltration; RFS: relapse-free survival.

\section{Acknowledgements}

The authors are extremely grateful to Prof. Alexander A. Stadnikov, the Head of Department of Histology, Cytology and Cell Biology of the Orenburg State Medical Academy for the help in the organization of this study.

\section{Competing Interests}

The authors have declared that no competing interest exists.

\section{References}

1. Folkman J. Is tissue mass regulated by vascular endothelial cells? Prostate as the first evidence. Endocrinology 1998; 139(2): 441-442. doi: $10.1210 /$ en.139.2.441.

2. Atkin GK, Chopada A. Tumour angiogenesis: the relevance to surgeons. Ann R Coll Surg Engl. 2006; 88: 525-529. doi: 10.1308/003588406X117106.

3. Sussman LK, Upalakalin JN, Roberts MJ, et al. Blood markers for vasculogenesis increase with tumor progression in patients with breast carcinoma. Cancer Biology Therapy 2003; 2: 255-256.

4. Baluk P, Morikawa S, Haskell A, et al. Abnormalities of Basement Membrane on Blood Vessels and Endothelial Sprouts in Tumors. American Journal of Pathology 2003; 163 (5): 1801 - 1815

5. Morikawa S, Baluk P, Kaidoh T, et al. Abnormalities in Pericytes on Blood Vessels and Endothelial Sprouts in Tumors. American Journal of Pathology 2002; 160(3): 985 - 1000.

6. Holash J, Maisonpierre PC, Compton D, et al. Vessel cooption, regression, and growth in tumors mediated by angiopoietins and VEGF. Science 1999; 5422: 1994-1998. DOI:10.1126/science.284.5422.1994.

7. Chang YS, di Tomaso E, McDonald DM, et al. Mosaic blood vessels in tumors: Frequency of cancer cells in contact with flowing blood. PNAS 2000; 97(26): 14608-14613.

8. Wang W, Lin P, Han C, et al. Vasculogenic mimicry contributes to lymph node metastasis of laryngeal squamous cell carcinoma. J Exp Clin Cancer Res. 2010; 29: 60. doi: 10.1186/1756-9966-29-60.

9. Burri PH, Hlushchuk R, Djonov V. Intussusceptive Angiogenesis: Its Emergence, Its Characteristics, and Its Significance. Developmental Dinamics 2004; 231: 474 - 478. DOI: $10.1002 /$ dvdy. 20184.

10. Asahara T, Masuda H, Takahashi T, et al. Bone marrow origin of endothelial cell progenitor cells responsible for postnatal vasculogenesis in physiological and pathological neovascularization. Circ Res. 1999; 85:221-228. doi: 10.1161/ 01.RES.85.3.221.

11. Sun B, Zhang S, Ni C, et al. Correlation between melanoma angiogenesis and the mesenchymal stem cells and endothelial progenitor cells derived from bone marrow. Stem Cells Dev. 2005; 14(3):292-8.

12. [No authors listed]. TNM Classification of Malignant Tumours -7 edition. http://www.UICC.org

13. Eiro N, Vizoso FJ. Inflammation and cancer. World J Gastrointest Surg. 2012; 4(3):62-72. doi: 10.4240 /wjgs.v4.i3.62.

14. Wu Y, Zhou BP. Inflammation: a driving force speeds cancer metastasis. Cell Cycle 2009; 8(20): 3267-3273.

15. De Narddo DG, Johansson M, Coussens LM. Immune cells as mediators of solid tumor metastasis. Cancer Metastasis Rev. 2008; 27:11-18. DOI: $10.1007 /$ s10555-007-9100-0.

16. Pollard JW. Trophic macrophages in development and disease. Nat Rev Immunol. 2009; 9(4):259-70. doi: 10.1038/nri2528.

17. Mantur M, Wojszel J. Cell adhesion molecules and their participation in the process of inflammation and cancerogenesis. Pol Merkur Lekarski 2008; 24(140):177-80.

18. Reiss K, Ludwig A, Saftig P. Breaking up the tie: disintegrin-like metalloproteinases as regulators of cell migration in inflammation and invasion. Pharmacol Ther. 2006; 111(3):985-1006.

19. Man Y.G. Aberrant leukocyte infiltration: a direct trigger for breast tumor invasion and metastasis. Int J Biol Sci. 2010; 6(2):129-32.

20. Man YG, Stojadinovic A, Mason J, et al. Tumor-Infiltrating Immune Cells Promoting Tumor Invasion and Metastasis: Existing Theories. J. Cancer 2013; 4(1):84-95. doi:10.7150/jca.5482.

21. Man YG, Harley R, Mason J, Gardner WA. Contributions of leukocytes to tumor invasion and metastasis: the "piggy-back" hypothesis. Cancer Epidem. 2010; 34:3-6.

22. Pawelek JM, Chakraborty AK. The cancer cell--leukocyte fusion theory of metastasis. Adv Cancer Res. 2008; 101:397-444. 
23. Acs G, Dumoff KL, Solin LJ, et al. Extensive Retraction Artifact Correlates With Lymphatic Invasion and Nodal Metastasis and Predicts Poor Outcome in Early Stage Breast Carcinoma. Am J Surg Pathol. 2007; 31:129-140.

24. Acs G, Paragh G, Rakosy Z, et al. The extent of retraction clefts correlates with lymphatic vessel density and VEGF-C expression and predicts nodal metastasis and poor prognosis in early-stage breast carcinoma. Mod Pathol. 2012; 25(2):163-177. doi: 10.1038/modpathol.2011.138.

25. Zaorsky NG, Patil N, Freedman GM, Tuluc M. Differentiating lymphovascular invasion from retraction artifact on histological specimen of breast carcinoma and their implications on prognosis. J Breast Cancer 2012; 15(4):478-80.

26. Man YG. Tumor cell budding from focally disrupted tumor capsules: a common pathway for all breast cancer subtype derived invasion?. J Cancer 2010; 1:32-37. doi:10.7150/jca.1.32.

27. Man YG. A seemingly most effective target for early detection and intervention of prostate tumor invasion. J Cancer 2010; 1:63-69. doi:10.7150/jca.1.63.

28. Nagy JA, Dvorak AM, Dvorak HF. Vascular hyperpermeability, angiogenesis, and stroma generation. Cold Spring Harb Perspect Med. 2012; 2(2):a006544. doi: $10.1101 /$ cshperspect.a006544.

29. van der Bilt JD, Borel Rinkes IH. Surgery and angiogenesis. Biochim Biophys Acta 2010; 1654:95-104. doi: 10.1016/j.bbcan.2004.01.003.

30. Mikasa K, Sawaki M, Kita E, et al. Significant survival benefit to patients with advanced non-small-cell lung cancer from treatment with clarithromycin. Chemotherapy 1997; 43(4):288-296.

31. Morris TC, Kettle PJ, Drake M, et al. Clarithromycin with low dose dexamethasone and thalidomide is effective therapy in relapsed/refractory myeloma. Br J Haematol. 2008; 143(3):349-54. DOI: 10.1111/j.1365-2141.2008.07360.x.

32. Sauter C, Blum S. Regression of lung lesions in Hodgkin's disease by antibiotics: case report and hypothesis on the etiology of Hodgkin's disease. Am J Clin Oncol. 2003; 26(1):92-4. 